\title{
Inhibitors of heat shock protein 90 augment endothelin-1-induced heat shock protein 27 through the SAPK/JNK signaling pathway in osteoblasts
}

\author{
KAZUHIKO FUJTAA ${ }^{1,2}$, TAKANOBU OTSUKA ${ }^{1}$, TETSU KAWABATA ${ }^{1,2}$, GO SAKAI $^{1,2}$, \\ RIE MATSUSHIMA-NISHIWAKI ${ }^{2}$, OSAMU KOZAWA ${ }^{2}$ and HARUHIKO TOKUDA ${ }^{2,3}$ \\ ${ }^{1}$ Department of Orthopedic Surgery, Nagoya City University Graduate School of Medical Sciences, Nagoya 467-8601; \\ ${ }^{2}$ Department of Pharmacology, Gifu University Graduate School of Medicine, Gifu 501-1194; \\ ${ }^{3}$ Department of Clinical Laboratory and Biobank, National Center for Geriatrics and Gerontology, Obu 474-8511, Japan
}

Received September 25, 2017; Accepted April 6, 2018

DOI: $10.3892 / \mathrm{mmr} .2018 .8878$

\begin{abstract}
It has been previously reported that endothelin-1 (ET-1) stimulates the induction of heat shock protein (HSP) 27 through the activation of $\mathrm{p} 38$ mitogen-activated protein (MAP) kinase and stress-activated protein kinase/c-Jun $\mathrm{N}$-terminal kinase (SAPK/JNK) in osteoblast-like MC3T3-E1 cells. The present study investigated whether HSP90, a high-molecular-weight HSP, was implicated in the ET-1-stimulated HSP27 induction in MC3T3-E1 cells. The effects of HSP90 inhibitors on the induction of HSP27 were examined. The HSP90 inhibitors geldanamycin and 17-demethoxygeldanamycin (17-DMAG) significantly amplified HSP27 induction stimulated by ET-1 in a dose-dependent manner. In addition, onalespib (another HSP90 inhibitor) significantly strengthened the ET-1-induced HSP27 protein levels. The ET-1-stimulated phosphorylation of p38 MAP kinase was minimally affected by geldanamycin, 17-DMAG or onalespib. Onalespib and 17-DMAG significantly enhanced the ET-1-induced phosphorylation of SAPK/JNK. In addition, SP600125, a SAPK/JNK inhibitor, notably reduced the amplification by onalespib of ET-1-induced HSP27. These results suggest that HSP90 limits ET-1-stimulated HSP27 induction at a point upstream of SAPK/JNK in osteoblasts. These results suggest that HSP90 may be a novel clinical target for metabolic bone diseases, including osteoporosis.
\end{abstract}

Correspondence to: Dr Osamu Kozawa, Department of Pharmacology, Gifu University Graduate School of Medicine, Yanagido 1-1, Gifu 501-1194, Japan

E-mail: okkasugai@yahoo.co.jp

Key words: heat shock protein 27, heat shock protein 90 inhibitor, endothelin-1, stress-activated protein kinase/c-Jun N-terminal kinase, osteoblast

\section{Introduction}

Heat shock proteins (HSPs) have been first discovered as stress-inducible proteins $(1,2)$. It is generally recognized that HSPs intracellularly act as molecular chaperones and regulate proteostasis under stress conditions $(1,2)$. Based on their molecular masses, HSPs are currently classified into seven families, including HSPA (HSP70), HSPB (small HSPs), HSPC (HSP90) and HSPH (HSP110) (3). Among small HSPs with monomer molecular mass in the range of $12-43 \mathrm{kDa}$, HSP27, an ATP-independent molecular chaperone, is induced by the heat shock associated with physical and chemical stresses, including radiation, oxidative stress and various chemotherapies (1). HSP27 is able to bind to improperly folded proteins and further transfer them to the ATP-dependent chaperones or to the protein degradation machines including proteasomes or autophagosomes. The functions of HSP27 are regulated by post-translational modifications such as phosphorylation (1). Unphosphorylated HSP27 forms large aggregated oligomers while its phosphorylation results in the conformational changes leading to dissociated small oligomers (1). On the other hand, HSP90, an ATP-dependent molecular chaperone, ubiquitously and abundantly exist in a variety of unstressed cells, represent $1-2 \%$ of total cellular proteins (2). In addition to protein folding, it is well known that HSP90 regulates the binding of glucocorticoid to its specific receptor under physiological conditions (2). Evidence is accumulating that HSPs are involved in a variety of pathophysiological cell processes $(1,2)$.

Bone metabolism is strictly regulated by two functional cells, osteoblasts and osteoclasts (4). The former cells contribute to bone formation, whereas the latter cells are responsible for bone resorption. In order to maintain the stability of bone and the integrity, bone tissue is constantly reconstructed by a sequential process consisted of the resorption of old bone and the subsequent formation of new bone, so called bone remodeling (5). Regarding HSP27 in osteoblasts, down-regulation of osteoblast proliferation is reportedly accompanied by a transient increase in the HSP27 mRNA expression (6). In addition, it has been shown that estrogen facilitates the induction of HSP27 stimulated by heat (7). We have previously reported 
that endothelin-1 (ET-1), a bone remodeling agent, stimulates the induction of HSP27 in osteoblast-like MC3T3-E1 cells and that p38 mitogen-activated protein (MAP) kinase and stress activated protein kinase/c-Jun N-terminal kinase (SAPK/JNK) play as positive regulators in the HSP27 induction $(8,9)$. In addition, we demonstrated that the mineralization of MC3T3-E1 cells is modulated by the expression level of HSP27 protein and its phosphorylation status (10). On the other hand, as for HSP90 in osteoblasts, the expression of HSP90 protein is reportedly induced by bisphosphonate, the most useful medicine for osteoporosis treatment (11). In addition, it has recently been shown that low-intensity pulsed ultrasound stimulation reportedly upregulates HSP90 level, leading to the formation of mineralized nodule (12). However, the details of both HSP27 and HSP90 in osteoblasts have not yet been clarified.

In the present study, we investigated the involvement of HSP90 in the ET-1-stimulated HSP27 induction in these cells. We herein show that HSP90 inhibitors amplify the ET-1-induced HSP27 protein levels in MC3T3-E1 cells and that the up-regulating effect of HSP90 inhibitors was exerted via SAPK/JNK.

\section{Materials and methods}

Materials. ET-1 was obtained from Peptide Institute Inc. (Minoh, Osaka, Japan). Geldanamycin was purchased from Sigma-Aldrich; Merck KGaA (Darmstadt, Germany). The inhibitors 17-demethoxygeldanamycin (17-DMAG) and SP600125 were obtained from EMD Millipore (Billerica, MA, USA). Onalespib was purchased from Selleck Chemicals (Houston, TX, USA). HSP27 antibodies and glyceraldehyde 3 phosphate dehydrogenase (GAPDH) antibodies were obtained from Santa Cruz Biotechnology, Inc., (Dallas, TX, USA). Phospho-specific p38 MAP kinase antibodies, p38 MAP kinase antibodies, phospho-specific SAPK/JNK antibodies and SAPK/JNK antibodies were purchased from Cell Signaling Technology, Inc., (Danvers, MA, USA). An ECL western blotting detection system was purchased from GE Healthcare Life Sciences (Little Chalfont, UK). Other materials and chemicals were obtained from commercial sources. Geldanamycin, 17-DMAG, onalespib and SP600125 were dissolved in dimethyl sulfoxide. The maximum concentration of dimethyl sulfoxide was $0.1 \%$, which did not affect the assay for western blot analysis.

Cell culture. Cloned osteoblast-like cells, MC3T3-E1 cells that have been derived from newborn mouse calvaria (13), were maintained as previously described (14). In brief, the cells were cultured in $\alpha$-minimum essential medium ( $\alpha$-MEM) containing $10 \%$ fetal bovine serum (FBS) at $37^{\circ} \mathrm{C}$ in a humidified atmosphere of $5 \% \mathrm{CO}_{2} / 95 \%$ air. The cells were seeded into $90-\mathrm{mm}$ diameter dishes $\left(20 \times 10^{4} / \mathrm{dish}\right)$ for western blot analysis. After 5 days, the medium was exchanged to $\alpha$-MEM containing $0.3 \%$ FBS. The cells were then used for experiments after $48 \mathrm{~h}$.

Western blot analysis. Western blotting was performed as described previously as follows (15). The cultured cells were pretreated with various doses of geldanamycin, 17-DMAG or onalespib for $60 \mathrm{~min}$, and then stimulated by $0.1 \mu \mathrm{M}$ ET- 1 or vehicle in the presence of inhibitors in $\alpha$-MEM containing $0.3 \%$ FCS for the indicated periods. The cells were washed twice with phosphate-buffered saline and then lysed, homogenized and sonicated in a lysis buffer containing $62.5 \mathrm{mM}$ Tris/ $\mathrm{HCl}, \mathrm{pH} 6.8$, $2 \%$ sodium dodecyl sulfate (SDS), $50 \mathrm{mM}$ dithiothreitol and $10 \%$ glycerol. SDS-polyacrylamide gel electrophoresis (PAGE) was performed by the method of Laemmli in $10 \%$ polyacrylamide gel (16). The protein was fractionated and transferred onto an Immun-Blot polyvinylidine difluoride (PVDF) membrane (BioRad Laboratories, Inc., Hercules, CA, USA). The membranes were blocked with $5 \%$ fat-free dry milk in Tris-buffered saline-Tween (TBS-T; $20 \mathrm{mM}$ Tris/ $\mathrm{HCl}, \mathrm{pH}$ 7.6, $137 \mathrm{mM}$ $\mathrm{NaCl}, 0.1 \%$ Tween-20) for $1 \mathrm{~h}$ before incubation with primary antibodies. Western blot analysis was performed using HSP27 antibodies, GAPDH antibodies, phospho-specific p38 MAP kinase antibodies, p38 MAP kinase antibodies, phospho-specific SAPK/JNK antibodies and SAPK/JNK antibodies with peroxidase-labeled antibodies raised in goat anti-rabbit IgG which were used as secondary antibodies. Peroxidase activity on PVDF membranes was visualized on X-ray film by means of the ECL western blotting detection system.

Densitometric analysis. A densitometric analysis of the western blots was performed using a scanner and image analysis software program (ImageJ v1.48; National Institutes of Health, Bethesda, MD, USA). HSP27 protein levels or the phosphorylated protein levels were calculated as follows: The background-subtracted intensity was respectively normalized to GAPDH intensity or the total protein intensity, respectively, and plotted as the fold increase in comparison to that of the control cells without stimulation.

Statistical analysis. All data are presented as the mean \pm SEM of triplicate determinations from three independent cell preparations. The data were analyzed by ANOVA followed by Bonferroni method for multiple comparisons between pairs. $\mathrm{P}<0.05$ was considered to indicate a statistically significant difference.

\section{Results}

Effects of geldanamycin, 17-DMAG or onalespib on ET-1-stimulated HSP27 induction in MC3T3-E1 cells. We have previously shown that ET-1 induces the expression of HSP27 protein in osteoblast-like MC3T3-E1 cells (8). In the present study, in order to clarify whether HSP90 is implicated in the ET-1-stimulated HSP27 induction in MC3T3-E1 cells, we examined the effects of HSP90 inhibitors on the HSP27 induction. Geldanamycin, an inhibitor of HSP90 (17), significantly enhanced the ET-1-induced levels of HSP27 protein (Fig. 1A). The effect of geldanamycin on the HSP27 induction was dose-dependent over the range between 10 and $30 \mathrm{nM}$ (Fig. 1A). We also found that 17-DMAG, a less toxic derivative of geldanamycin (18), dose dependently augmented the ET-1-stimulated HSP27 induction in the range between 10 and $30 \mathrm{nM}$ (Fig. 1B).

In addition, onalespib, a HSP90 inhibitor, which is a different type from geldanamycin (19), as well as geldanamycin and its derivative, markedly upregulated the HSP27 protein levels induced by ET-1 (Fig. 1C). 

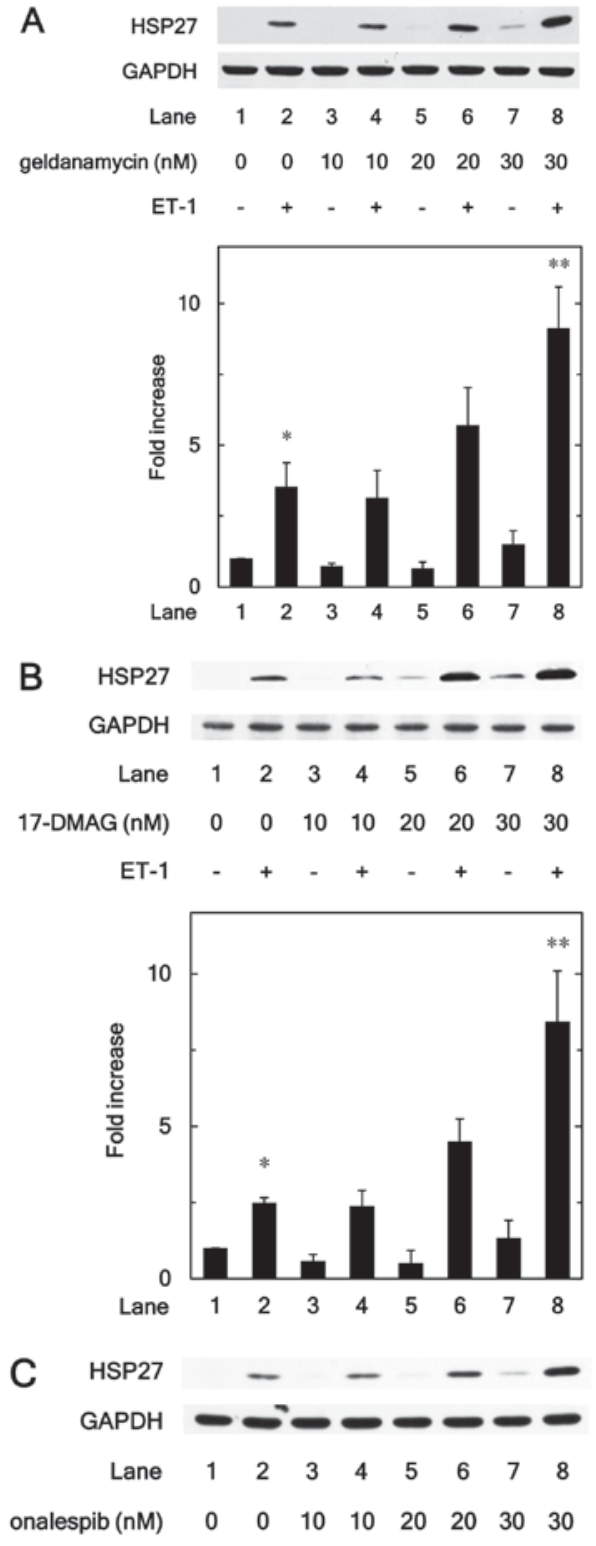

$\mathrm{ET}-1-+-+++$

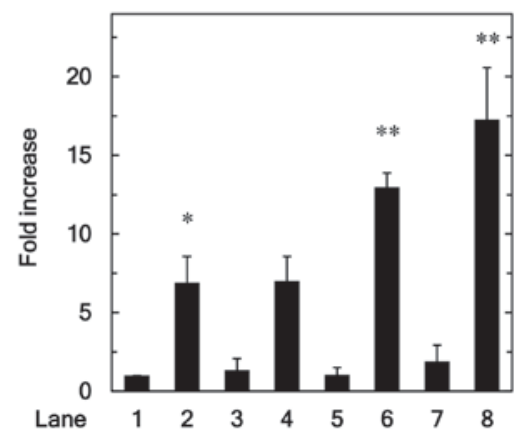

Figure 1. Effects of geldanamycin, 17-DMAG or onalespib on ET-1-stimulated HSP27 induction in MC3T3-E1 cells. The cultured cells were pretreated with various doses of (A) geldanamycin, (B) 17-DMAG or (C) onalespib for $60 \mathrm{~min}$ and then stimulated by $0.1 \mu \mathrm{M}$ ET- 1 or vehicle for $9 \mathrm{~h}$. The extracts of cells were subjected to SDS-PAGE with subsequent western blot analysis with antibodies against HSP27 or GAPDH. The histogram indicates the quantitative representations of the levels of HSP27 following normalization with respect to those of GAPDH obtained from laser densitometric analysis. The levels were expressed as the fold increase to the basal levels presented in lane 1. Each value represents the mean + standard error of the mean of triplicate determinations from three independent cell preparations. ${ }^{*} \mathrm{P}<0.05$ vs. the control (lane 1). ${ }^{* *} \mathrm{P}<0.05$ vs. ET-1 alone (lane 2). ET-1, endothelin-1; HSP, heat shock protein; 17-DMAG, 17-demethoxygeldanamycin.
Effects of geldanamycin, 17-DMAG or onalespib on the ET-1-induced phosphorylation of p38 MAP kinase in MC3T3-E1 cells. In our previous study (8), we have reported that p38 MAP kinase acts as a positive intracellular molecule in the ET-1-stimulated HSP27 induction in osteoblast-like MC3T3-E1 cells. In order to investigate whether the HSP90 inhibitor-effect on the ET-1-stimulated HSP27 induction is dependent on the activation of p38 MAP kinase in these cells, we examined the effects of geldanamycin, 17-DMAG or onalespib on the ET-1-induced phosphorylation of p38 MAP kinase. However, geldanamycin (Fig. 2A), 17-DMAG (Fig. 2B) or onalespib (Fig. 2C) failed to affect the ET-1-induced phosphorylation levels of p38 MAP kinase.

Effects of onalespib or 17-DMAG on the ET-1-induced phosphorylation of SAPK/JNK in MC3T3-E1 cells. We have demonstrated that SAPK/JNK in addition to p38 MAP kinase is involved in the ET-1-stimulated HSP27 induction in MC3T3-E1 cells (9). Thus, we next examined the effect of onalespib on the ET-1-induced phosphorylation of SAPK/JNK. Onalespib, which alone did not affect the SAPK/JNK phosphorylation, significantly strengthened the ET-1-induced phosphorylation levels of SAPK/JNK (Fig. 3A). The amplifying effect of onalespib on the SAPK/JNK phosphorylation was dose-dependent over the range between 0.3 and $1.0 \mu \mathrm{M}$ (Fig. 3A). Additionally, we showed that 17-DMAG remarkably enhanced the ET-1-induced levels of phosphorylated SAPK/JNK (Fig. 3B).

Effect of SP600125 on the enhancement by onalespib of ET-1-stimulated HSP27 induction in MC3T3-E1 cells. In order to further investigate whether HSP90 inhibitor enhances the ET-1-stimulated HSP27 induction via SAPK/JNK activated by ET-1 in MC3T3-E1 cells, we examined effect of SP600125, an inhibitor of SP600125 (20), on enhancement by onalespib of ET-1-induced HSP27. SP600125 significantly reduced the amplification by onalespib of ET-1-induced levels of HSP27 protein (Fig. 4).

\section{Discussion}

In the present study, we showed that geldanamycin and 17-DMAG, a geldanamycin derivative, which belong to HSP90 inhibitors $(17,18)$, significantly potentiated the ET-1-stimulated induction of HSP27 (HSPB1), a small HSP (HSPB), in osteoblast-like MC3T3-E1 cells. Furthermore, onalespib (19), another HSP90 inhibitor different from geldanamycin or its analogues, markedly increased the ET-1-induced HSP27 protein levels. Based on our findings, it seems likely that HSP90 plays a suppressive role in ET-1-stimulated HSP27 induction in osteoblast-like MC3T3-E1 cells.

Regarding the intracellular signaling of ET-1 in osteoblasts, we have demonstrated that ET-1 stimulates the induction of HSP27 via p38 MAP kinase and SAPK/JNK among the MAP kinase superfamily (21), in osteoblast-like MC3T3-E1 cells $(8,9)$. Based on our findings, we investigated whether the amplifying effect of HSP90 inhibitors on the ET-1-stimulated HSP27 induction is due to the modulation of these MAP kinases in MC3T3-E1 cells. However, the 

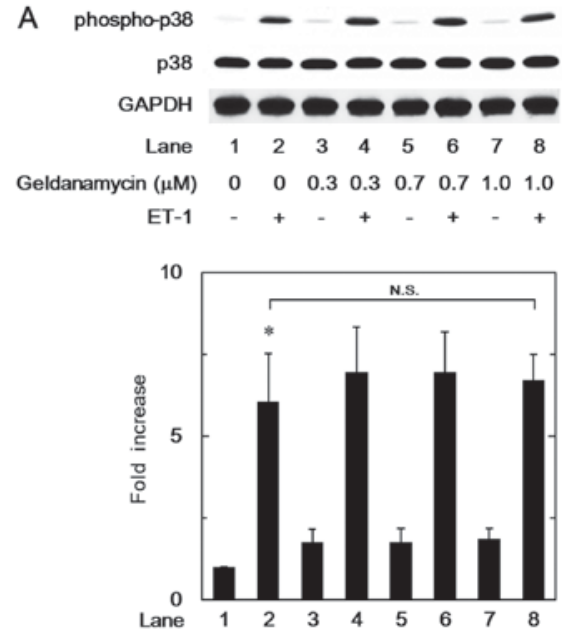

B
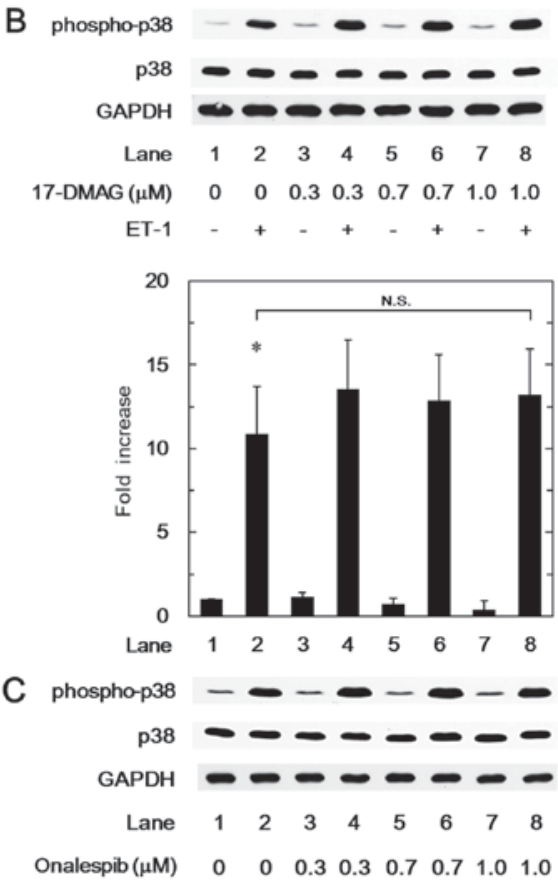

$\mathrm{ET}-1-++++$

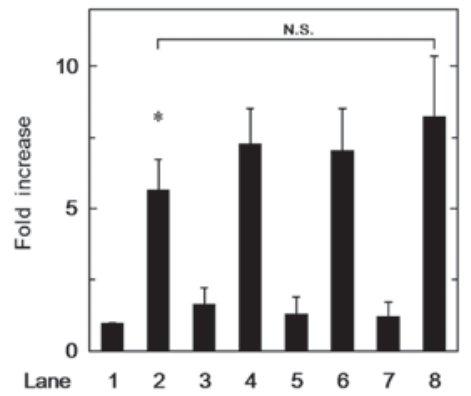

Figure 2. Effects of geldanamycin, 17-DMAG or onalespib on ET-1-induced phosphorylation of p38 MAP kinase in MC3T3-E1 cells. The cultured cells were pretreated with various doses of (A) geldanamycin, (B) 17-DMAG or (C) onalespib for $60 \mathrm{~min}$ and then stimulated by $0.1 \mu \mathrm{M}$ ET-1 or vehicle for $20 \mathrm{~min}$. The extracts of cells were subjected to SDS-PAGE with subsequent western blot analysis with antibodies against phospho-specific p38 MAP kinase or p38 MAP kinase. The histogram shows the quantitative representations of the levels of phosphorylated p38 MAP kinase after normalization with respect to those of p38 MAP kinase obtained from laser densitometric analysis. The levels were expressed as the fold increase to the basal levels presented in lane 1. Each value represents the mean + standard error of the mean of triplicate determinations from three independent cell preparations. ${ }^{*} \mathrm{P}<0.05$ vs. the control (lane 1). N.S, no significant difference; ET-1, endothelin-1; 17-DMAG, 17-demethoxygeldanamycin; phospho, phosphorylated; MAP, mitogen-activated protein.
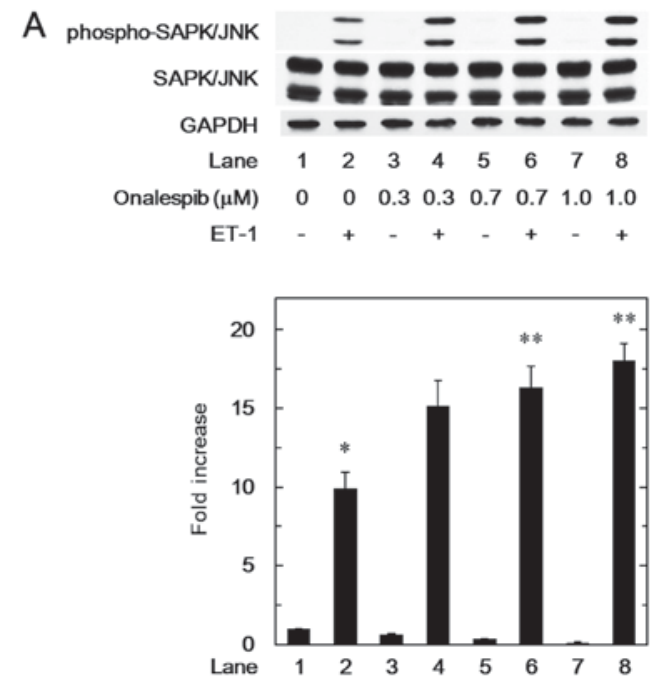

B
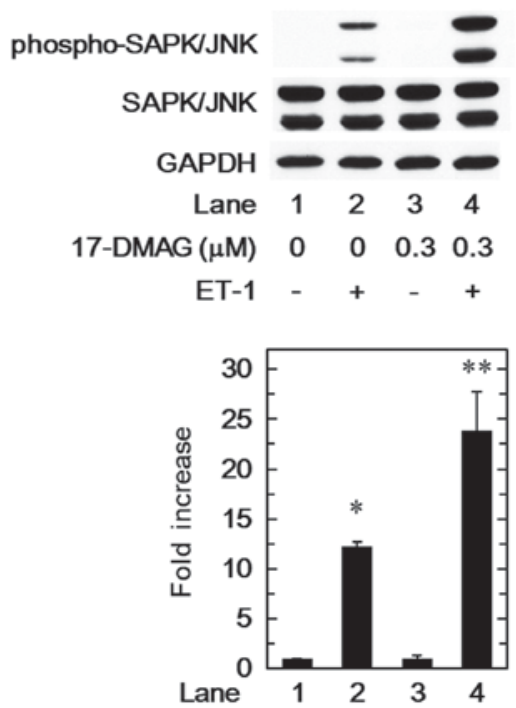

Figure 3. Effects of onalespib or 17-DMAG on the ET-1-induced phosphorylation of SAPK/JNK in MC3T3-E1 cells. The cultured cells were pretreated with various doses of (A) onalespib or (B) 17-DMAG for $60 \mathrm{~min}$ and then stimulated by $0.1 \mu \mathrm{M}$ ET-1 or vehicle for $30 \mathrm{~min}$. The extracts of cells were subjected to SDS-PAGE with subsequent western blot analysis with antibodies against phospho-specific SAPK/JNK or SAPK/JNK. The histogram shows the quantitative representations of the levels of phospho-SAPK/JNK following normalization with respect to those of SAPK/JNK obtained from laser densitometric analysis. The levels were expressed as the fold increase to the basal levels presented as lane 1. Each value represents the mean + standard error of the mean of triplicate determinations from three independent cell preparations. ${ }^{*} \mathrm{P}<0.05$ vs. the control (lane 1$)$. ${ }^{* *} \mathrm{P}<0.05$ vs. ET-1 alone (lane 2 ). ET-1, endothelin-1; 17-DMAG, 17-demethoxygeldanamycin; phospho, phosphorylated; SAPK, stress-activated protein kinase; JNK, c-Jun N-terminal kinase.

HSP90 inhibitors, geldanamycin, 17-DMAG and onalespib, did not have significant effects on the ET-1-induced levels of phosphorylated p38 MAP kinase. Therefore, it seems unlikely that HSP90 affects the ET-1-stimulated HSP27 induction via the signaling pathway of p38 MAP kinase in osteoblast-like MC3T3-E1 cells. On the other hand, we showed that onalespib significantly augmented the ET-1-induced phosphorylated levels of SAPK/JNK. In addition, the SAPK/JNK phosphorylation induced by ET-1 was markedly enhanced by 17-DMAG. Furthermore, we demonstrated that the enhancement by onalespib of the 

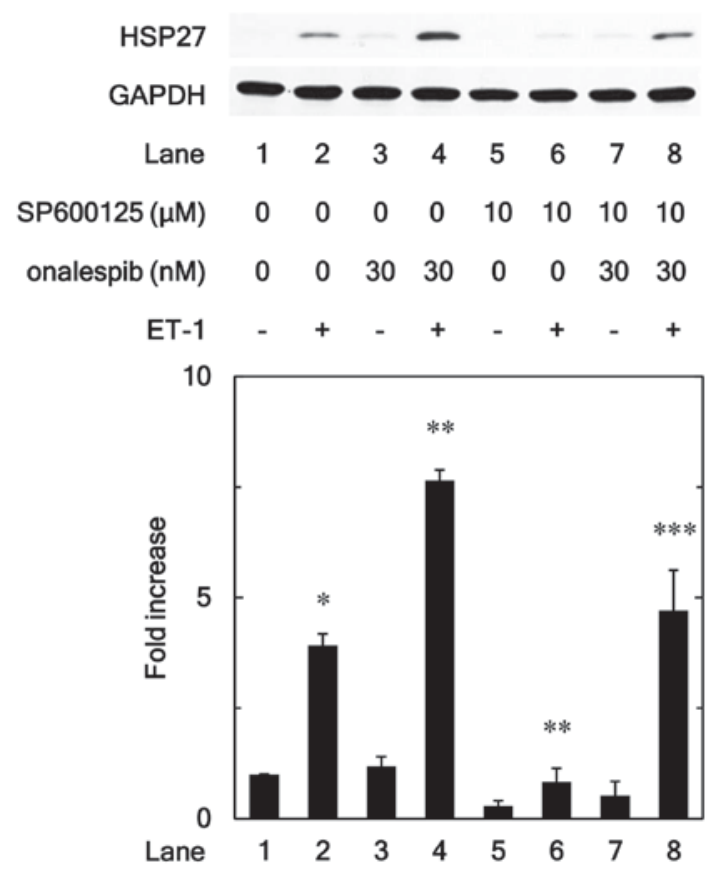

Figure 4. Effect of SP600125 on amplification by onalespib of ET-1-stimulated HSP27 induction in MC3T3-E1 cells. The cultured cells were pretreated with $10 \mu \mathrm{M}$ SP600125 or vehicle for $60 \mathrm{~min}$. The cells were then preincubated by $30 \mathrm{nM}$ onalespib or vehicle for $60 \mathrm{~min}$ and stimulated by $0.1 \mu \mathrm{M}$ ET- 1 or vehicle for $9 \mathrm{~h}$. The extracts of cells were subjected to SDS-PAGE with subsequent western blot analysis with antibodies against HSP27 or GAPDH. The histogram shows the quantitative representations of the levels of HSP27 after normalization with respect to those of GAPDH obtained from laser densitometric analysis. The levels were expressed as the fold increase to the basal levels presented as lane 1. Each value represents the mean + standard error of the mean of triplicate determinations from three independent cel preparations. ${ }^{*} \mathrm{P}<0.05$ vs. the control (lane 1). ${ }^{* *} \mathrm{P}<0.05$ vs. ET-1 alone (lane 2 ) ${ }^{* * *} \mathrm{P}<0.05$ vs. ET-1 with onalespib preincubation (lane 4). ET-1, endothelin-1; HSP, heat shock protein.

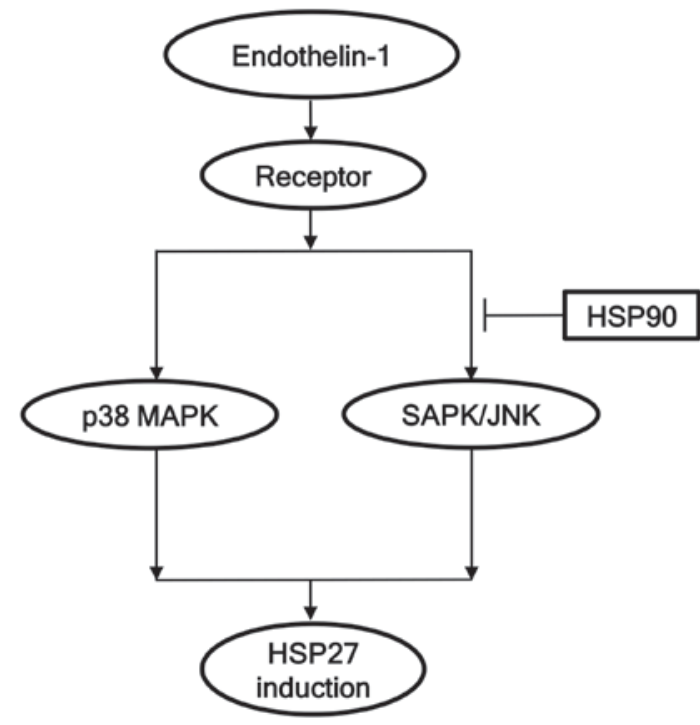

Figure 5. Schematic illustration of the regulatory mechanism of HSP90 in the ET-1-stimulated HSP27 induction in osteoblasts. ET-1, endothelin-1; HSP, heat shock protein; p38 MAPK, p38 mitogen-activated protein kinase; SAPK, stress-activated protein kinase; JNK, c-Jun N-terminal kinase.

ET-1-stimulated HSP27 induction was reduced by an inhibitor of SAPK/JNK, SP600125 (20). Taking our findings into account as a whole, it is most likely that HSP90 acts at a point upstream from SAPK/JNK and negatively regulates the ET-1-stimulated HSP27 induction in osteoblast-like MC3T3-E1 cells. The potential mechanism of HSP90 in the ET-1-induced HSP27 in osteoblasts shown here is summarized in Fig. 5.

It is firmly established that HSP90, a major molecular chaperone, plays as a central regulator of proteostasis such as protein folding under stress conditions (2). In addition to protein folding, accumulating evidence indicates that HSP90 is implicated in a variety of physiological and pathological cell processes including responses to steroid hormones, and neurodegenerative diseases (2). Regarding HSP90 in osteoblasts, it has been shown that bisphosphonate, the most useful medicine for osteoporosis, stimulates expression of HSP90 in osteoblasts (11). In addition, low-intensity pulsed ultrasound stimulation reportedly enhances osteoblasts proliferation and up-regulates HSP90, leading to dense mineralization (12). We have previously demonstrated that phosphorylated HSP27 acts as a negative regulator of calcification in osteoblast-like MC3T3-E1 cells whereas un-phosphorylated HSP27 has a stimulatory effect on the mineralization (10). It is well known that the functions of small HSPs are regulated by post-translational modifications including phosphorylation, indicating that phosphorylated status of HSP27 has a switching role in cellular functions (1). Our present results show that HSP90 inhibitors limits the ET-1-stimulated HSP27 induction in osteoblast-like MC3T3-E1 cells. Based on these findings, it seems likely that HSP90 regulates the mineralization of osteoblasts through modulating the HSP27 protein levels. Our present findings regarding about the regulation by HSP90 inhibitors of the HSP27 induction in osteoblasts might provide a new aspect of HSP90 as a therapeutic target for metabolic bone diseases such as osteoporosis. However, the physiological significance of HSP27 in osteoblasts remains unclear. Further investigations using another human osteoblast cells such as USO2 or MG63 would be required to clarify the exact roles of HSP90 and HSP27 in bone metabolism.

In conclusion, our results strongly suggest that HSP90 negatively regulates ET-1-stimulated HSP27 induction at a point upstream of SAPK/JNK in osteoblasts.

\section{Acknowledgements}

The authors would like to thank Yumiko Kurokawa for her skillful technical assistance.

\section{Funding}

The present study was funded by Grant-in-Aid for Scientific Research (grant nos. 26462289, 15K10487, 17K11002) from the Ministry of Education, Culture, Sports, Science and Technology of Japan, and the Research Funding for Longevity Sciences (grant no. 26-12, 28-9) from National Center for Geriatrics and Gerontology, Japan.

\section{Availability of data and materials}

The datasets used and/or analyzed during the current study are available from the corresponding author on reasonable request. 


\section{Authors' contributions}

$\mathrm{KF}$, TO and OK conceived and designed the experiments. KF, TK, GS and RMN performed the experiments. KF, RMN, OK and HT analyzed the data. KF, TO, OK and HT wrote the paper. All authors read and approved the final manuscript.

\section{Ethics approval and consent to participate}

Not applicable.

\section{Consent for publication}

Not applicable.

\section{Competing interests}

The authors declare that they have no competing interests.

\section{References}

1. Mymrikov EV, Seit-Nebi AS and Gusev NB: Large potentials of small heat shock proteins. Physiol Rev 91: 1123-1159, 2011.

2. Schopf FH, Biebl MM and Buchner J: The HSP90 chaperone machinery. Nat Rev Mol Cell Biol 18: 345-360, 2017.

3. Kampinga HH, Hageman J, Vos MJ, Kubota H, Tanguay RM, Bruford EA, Cheetham ME, Chen B and Hightower LE: Guidelines for the nomenclature of the human heat shock proteins. Cell Stress Chaperones 14: 105-111, 2009.

4. Kular J, Tickner J, Chim SM and Xu J: An overview of the regulation of bone remodelling at the cellular level. Clin Biochem 45: 863-873, 2012.

5. Sims NA and Gooi JH: Bone remodeling: Multiple cellular interactions required for coupling of bone formation and resorption. Semin Cell Dev Biol 19: 444-451, 2008.

6. Shakoori AR, Oberdorf AM, Owen TA, Weber LA, Hickey E, Stein JL, Lian JB and Stein GS: Expression of heat shock genes during differentiation of mammalian osteoblasts and promyelocytic leukemia cells. J Cell Biochem 48: 277-287, 1992.

7. Cooper LF and Uoshima K: Differential estrogenic regulation of small M(r) heat shock protein expression in osteoblasts. J Biol Chem 269: 7869-7873, 1994.

8. Kawamura H, Otsuka T, Matsuno H, Niwa M, Matsui N, Kato K, Uematsu T and Kozawa O: Endothelin-1 stimulates heat shock protein 27 induction in osteoblasts: Involvement of p38 MAP kinase. Am J Physiol 277: E1046-E1054, 1999.
9. Tokuda $\mathrm{H}$, Niwa $\mathrm{M}$, Ito $\mathrm{H}$, Oiso $\mathrm{Y}$, Kato $\mathrm{K}$ and Kozawa $\mathrm{O}$ : Involvement of stress-activated protein kinase/c-Jun N-terminal kinase in endothelin-1-induced heat shock protein 27 in osteoblasts. Eur J Endocrinol 149: 239-245, 2003.

10. Kato K, Adachi S, Matsushima-Nishiwaki R, Minamitani C, Natsume H, Katagiri Y, Hirose Y, Mizutani J, Tokuda H, Kozawa $\mathrm{O}$ and Otsuka T: Regulation by heat shock protein 27 of osteocalcin synthesis in osteoblasts. Endocrinology 152: 1872-1882, 2011.

11. Romanello M, Bivi N, Pines A, Deganuto M, Quadrifoglio F, Moro L and Tell G: Bisphosphonates activate nucleotide receptors signaling and induce the expression of Hsp90 in osteoblast-like cell lines. Bone 39: 739-753, 2006.

12. Miyasaka M, Nakata H, Hao J, Kim YK, Kasugai S and Kuroda S: Low-intensity pulsed ultrasound stimulation enhances heat-shock protein 90 and mineralized nodule formation in mouse calvaria-derived osteoblasts. Tissue Eng Part A 21: 2829-2839, 2015.

13. Sudo H, Kodama H, Amagai Y, Yamamoto S and Kasai S: In vitro differentiation and calcification in a new clonal osteogenic cell line derived from newborn mouse calvaria. J Cell Biol 96: 191-198, 1983.

14. Kozawa O, Suzuki A, Tokuda H and Uematsu T: Prostaglandin F2alpha stimulates interleukin- 6 synthesis via activation of PKC in osteoblast-like cells. Am J Physiol 272: E208-E211, 1997.

15. Kato K, Ito H, Hasegawa $\mathrm{K}$, Inaguma Y, Kozawa $\mathrm{O}$ and Asano T: Modulation of the stress-induced synthesis of hsp27 and alpha B-crystallin by cyclic AMP in C6 rat glioma cells. J Neurochem 66: 946-950, 1996.

16. Laemmli UK: Cleavage of structural proteins during the assembly of the head of bacteriophage T4. Nature 227: 680-685, 1970.

17. Ochel HJ, Eichhorn K and Gademann G: Geldanamycin: The prototype of a class of antitumor drugs targeting the heat shock protein 90 family of molecular chaperones. Cell Stress Chaperones 6: 105-112, 2001.

18. Jez JM, Chen JC, Rastelli G, Stroud RM and Santi DV: Crystal structure and molecular modeling of 17-DMAG in complex with human Hsp90. Chem Biol 10: 361-368, 2003.

19. Ferraldeschi R, Welti J, Powers MV, Yuan W, Smyth T, Seed G, Riisnaes R, Hedayat S, Wang H, Crespo M, et al: Second-generation HSP90 inhibitor onalespib blocks mRNA splicing of androgen receptor variant 7 in prostate cancer cells. Cancer Res 76: 2731-2742, 2016.

20. Bennett BL, Sasaki DT, Murray BW, O'Leary EC, Sakata ST, Xu W, Leisten JC, Motiwala A, Pierce S, Satoh Y, et al: SP600125, an anthrapyrazolone inhibitor of Jun N-terminal kinase. Proc Natl Acad Sci USA 98: 13681-13686, 2001.

21. Kyriakis JM and Avruch J: Mammalian MAPK signal transduction pathways activated by stress and inflammation: A 10-year update. Physiol Rev 92: 689-737, 2012. 